\title{
EuroRxiv: toward a European open science repository
}

Khaled Moustafa

khaled.moustafa@eurorxiv.eu

Open and free access to scientific knowledge keeps scientists up to date with the latest achievements in their respective fields and to help set up appropriate solutions to health, environmental and technical issues. One of the efficient settings toward this purpose is the use of preprint servers- open repositories that allow authors to post their manuscripts ahead of formal peer review/publishing in traditional journals. The recognition of preprints as an essential part of science landscape are on the rise worldwide.

In 2018, a European funder coalition, called Coalition S, has been formed and issued an open access plan, called Plan $S$, that requires authors of studies funded by the Coalition to publish their manuscripts- starting from January 2021- in open access journals or repositories that meet the guidelines of the Plan S. Many publishers and researchers welcomed the Plan S as a step forward to promote openness and free access to publicly funded research.

To further enhance the open and free science movement, I'd propose a European preprint server called "European arXiv" (https://eurorxiv.eu) as a multidisciplinary and multilingual repository that will accept manuscripts (preprints and postprints) in the various European languages and beyond. The project is an individual initiative, but interested people are welcome to join. 